\title{
SENSITIVITY, RESOLUTION AND AMBIGUITY OF THE CRS STACK OPERATOR
}

\author{
Lourenildo W.B. Leite and Wildney W.S. Vieira
}

\begin{abstract}
This paper describes an investigation about the sensitivity and ambiguity of the Common Reflection Surface (CRS) stack operator parameters $\left(v_{0}, R_{\mathrm{NIP}}, R_{\mathrm{N}}, \alpha_{0}\right)$, and their resolution in terms of statistical properties of the solution of a nonlinear multi-parametric optimization problem for surface fitting between the forward model and a synthetic data, in the least-square sense. The sensitivity method is borrowed from dynamic system analysis and synthesis, and the definitions are based on the Miller-Murray model. The results are analyzed in terms of the CRS attributes search strategies during the stack process. The investigation principle is to combine global and local optimization methods to reach a minimum of the object function of minimization, where the problem matrix has a better linear relation to the parameters. A first search for a minimum is performed with a controlled random search method, followed by a gradient method for the last steps fo the optimization to calculate the data and parameter resolution and covariance matrices, and any further model statistical properties. The sensitivity functions are represented by the columns of the optimization problem matrix, and they in general exhibit a linear behavior instead of a convex form; as a result, this linear behavior establish the necessity of a good starting point for the optimized multi-parametric attributes search.
\end{abstract}

Keywords: CRS attributes, sensitivity analysis, resolution, ambiguity.

RESUMO. Este trabalho descreve uma investigação sobre a sensitividade e ambiguidade dos parâmetros $\left(v_{0}, R_{\mathrm{NIP}}, R_{\mathrm{N}}, \alpha_{0}\right)$ do operador de empilhamento CRS, e suas resoluções em termos das propriedades estatísticas da solução de um problema não-linear multi-paramétrico do ajuste da superfície de um modelo direto à de dados sintéticos, no sentido do quadrados-mínimos. 0 método da sensitividade é adaptado da análise e síntese de sistemas dinâmicos, e as definições são baseadas no modelo Miller-Murray. Os resultados são analisados em termos das estratégias de busca dos atributos CRS durante o processo de empilhamento. 0 princípio da investigação é combinar métodos de otimização global e local para alcançar um mínimo da função objeto de minimização, onde a matriz do problema tem uma melhor relação linear com os parâmetros. A primeira busca de um mínimo é realizada com um método de busca aleatória controlada, seguida por um método do gradiente para os últimos passos da otimização para calcular as matrizes resolução e covariância dos dados e parâmetros, e quaisquer propiedades estatísticas do modelo. As funções sensitividade são representadas pelas colunas da matriz otimização do problema, e em geral elas exibem um comportamento linear em vez de uma forma convexa; como resultado, este comportamento linear estabelece a necessidade de um bom ponto inicial para a busca multi-paramétrica otimizada dos atributos.

Palavras-chave: atributos CRS, análise de sensibilidade, resolução, ambiguidade. 


\section{INTRODUCTION}

The motivations of the present work come from two aspects of the CRS (Common Reflection Surface) stack: $\left(1^{\text {st }}\right)$ the presence of noise in the recovered attributes; and $\left(2^{\text {nd }}\right)$ the strategy for the parameter search. The analysis of the CRS outputs (stack, coherence, migration) show that as the observed input data improves on the signal/noise ratio, the attribute sections show a structure that resembles more the stack section that is used as reference. Sensitivity analysis can be used to determine how sensitive the model is to changes in the value of the parameters and to the geometry of the model, as part of a curve (CRS operator surface) fitting problem.

The present description is structured in three main parts related to curve fitting between the synthetic data and the forward model represented by the CRS stack operator, as an optimization problem in the least-square sense. The parts are: $\left(1^{\text {st }}\right)$ controlled random global search for the parameters; $\left(2^{\text {nd }}\right)$ second order gradient method, resolution and ambiguity; and $\left(3^{\text {rd }}\right)$ sensitivity analysis and its relation to the CRS attributes search.

Sensitivity and ambiguity calculus can be performed in the forward model as a first step in the analysis of the data fitting problem, and it is independent of the object function and of the mathematical optimization problem. This calculus is used for the purpose of analysis and synthesis of mathematical models. To be able to give a unique formulation of the mathematical problem, the mathematical model is usually considered to be known exactly, but this assumption is unrealistic since there is always a certain discrepancy between the actual system (data) and its mathematical model (operator). This discrepancy results from the following partial reasons, as we paraphrase Saltelli et al. (2004):

- A real system cannot be identified exactly because of the restricted accuracy of the measuring devices;

- Mathematical models are often simplified or idealized intentionally to simplify the mathematical problem, or to make it solvable at all.

For these reasons, the results of mathematical synthesis need not necessarily be practicable, or they may even be very poor, if there is considerable deviations between the real system and the mathematical model, and the solution be very sensitivity to the parameters. Therefore, it should be part of the practical problem to learn about parameter sensitivity prior to its implementation, or to reduce the sensitivity systematically if this turns out to be necessary.

This is important if one is involved in optimization procedures, since a natural property of optimization is to extremize the performance of a certain parameter set that controls the operation. Example of this are gradient methods, adaptative and selflearning systems.

Among the specific aims of the present study are: (1) to analyze the sensitivity of the CRS hyperbolic stack operator to its parameters; and (2) to compare the results of the parameter sensitivities with the strategy for the CRS attribute search as described by Muller (1999) and Mann (2002).

For the sensitivity analysis here discussed, we organized it as a nonlinear optimization problem, with the statistical properties obtained from the derivatives forming the problem matrix in the gradient method. The norm-2 was selected due to its simplicity and elegance, and we follow Tarantola (1984) for its description. Data and parameter resolution matrices, and the unit covariance matrix are calculated and analyzed at the object function minimum, where the problem is considered linearized based on Taylor series expansion to the first order.

The CRS parameter search strategies in the Semblance domain can be divided in two main parts: $\left(1^{\text {st }}\right)$ the first part is a search to determine initial parameter values to start the optimization iterations; $\left(2^{\text {nd }}\right)$ a second part is a simultaneous search for all parameters starting from the initial values. The searches are performed in the CMP (common-mid-point), therefore subject to properties of this family, as the reciprocity principle, and the aperture.

The forward model is represented by the CRS stack operator in terms of $t\left(x_{m}, h ; \mathbf{m}\right)$, where the independent variables are the mid-point $x_{m}$ and the source-sensor offset $h$, and $\mathbf{m}$ standing for the parameters represented by the attributes $\mathbf{m}=\left(R_{\mathrm{NIP}}, R_{\mathrm{N}}\right.$, $\left.\alpha_{0}\right)$, with $v_{0}$ considered fixed.

The optimization problem is presented in the following form: Given an observed seismic section $t^{(\text {obs })}\left(x_{m}, h\right)$ in the data space $D$, we wish to find a model $\mathbf{m}$ in the parameter space $M$ such that the forward model $t^{(\mathrm{pre})}\left(x_{m}, h ; \mathbf{m}\right)$ fits the observed data in the least-square sense.

The problem is classified as nonlinear, multiparametric, overdetermined. The synthetic data ca be constructed with different noise levels, and to avoid local minimum, a solution is constructed by the combination of the Controlled Random Search Global Method (CRSGM), followed by the Gradient Method (GM) to allow for the statistical analysis of the solution (Fletcher, 2001). The combination of these methods follows the principle that CRSGM defines a region around the global minimum, theoretically well defined, followed by last iterations around a local minimum with better linear properties to construct the correlation and resolution matrices. 
Numerical experiments were realized for randomly chosen points $P_{0}\left(x_{0}, t_{0}\right)$, therefore, neither to extend along the time traces, $\left(t_{0}=0, t_{\text {total }}\right)$, nor along the mid-points $\left(x_{0}=0\right.$, $\left.x_{\text {total }}\right)$, what would take the problem to be non-practicable. As established, the solution by the CRSGM can be used as an automatic input to the GM method, but in the present work these experiments were performed in two separate steps without degrading the strategy.

The structure of the sensitivity analysis is based on the polynomial function of the CRS operator represented by $t\left(x_{m}, h\right.$; $\mathbf{m})$, where now $\mathbf{m}=\left(v_{0}, R_{\mathrm{NIP}}, R_{\mathrm{N}}, \alpha_{0}\right)$, including $v_{0}$, and the quantitative properties of this system with respect to the $\mathbf{m}$ parameters are shown as functions of the independent variables $x_{m}$ and $h$.

The methodology for sensitivity analysis has been borrowed from the analysis and design of dynamic systems, as described, e.g., by Frank (1978) for engineering applications, and by many others for partial differential equations, e.g., Saltelli et al. (2004). Among the several methods for uncertainty and sensitivity analysis, the method adopted here is the local method which is derivative based.

The definitions to quantify the parameter sensitivity of a system is summarized later in the text, and we start defining the forward model to adjust the classical nomenclature to our subject.

\section{FORWARD MODEL}

The CRS stack operator describes the impulsive traveltime for curved reflectors based on the paraxial ray theory, and takes into account only primary reflection trajectories (Mann, 2002). The paraxial ray theory is based on Taylor series that handles general smooth functions of many variables. The quadratic model obtained by the truncated Taylor series of $t\left(x_{m}, h ; \mathbf{m}\right)$ about $\left(x_{m}=x_{0}, h=h_{0}\right)$ is denominated the parabolic traveltime for 3D, that can be written as:

$$
t\left(x_{m}, h ; \mathbf{m}\right)=t_{0}(\mathbf{m})+\mathbf{p}^{\top} \Delta \mathbf{x}+\frac{1}{2} \Delta \mathbf{x}^{\top} \mathbf{M} \Delta \mathbf{x} .
$$

The transit time $t\left(x_{m}, h ; \mathbf{m}\right)$ is calculated with respect to a central reference ray with traveltime $t_{0}\left(x_{0}, h_{0} ; \mathbf{m}\right)$, usually taken as the Normal Incidence Ray. Making $x_{1}=x_{m}$ and $x_{2}=h$, $\Delta x_{1}=x_{m}-x_{0}, \Delta x_{2}=h-h_{0}$, the Gradient $\mathbf{p}$ and the Hessian $\mathbf{M}$ are written as:

$$
\begin{gathered}
\mathbf{p}^{\top} \Delta \mathbf{x}=\sum_{n=1}^{2} \Delta x_{n} \frac{\partial}{\partial x_{n}} t\left(x_{m}, h ; \mathbf{m}\right) ; \\
\frac{1}{2} \Delta \mathbf{x}^{\top} \mathbf{M} \Delta \mathbf{x}=\frac{1}{2} \sum_{l=1}^{l=2} \sum_{n=1}^{2} \Delta x_{l} \Delta x_{n} \frac{\partial^{2}}{\partial x_{l} \partial x_{n}} t\left(x_{m}, h ; \mathbf{m}\right) .
\end{gathered}
$$

These equations also have time derivatives with respect to the survey space coordinates, where $\mathbf{p}$ and $\mathbf{M}$ can be calculated by Dynamic Ray Tracing.

The CRS operator can be physically interpreted by the $\mathrm{N}$ and NIP waves as shown in Figure 1. Duveneck (2004), among others, describe the transformation of Eq. (1) in terms of wave front parameters for 3D. But, limiting the discussion to the CRS2D, flat observation surface, and using the relations:

$$
\begin{gathered}
p=\frac{\sin \alpha_{0}}{v_{0}}, \quad M_{\mathrm{N}}=\frac{\cos ^{2} \alpha_{0}}{v_{0}} K_{\mathrm{N}} \quad \text { and } \\
M_{\mathrm{NIP}}=\frac{\cos ^{2} \alpha_{0}}{v_{0}} K_{\mathrm{NIP}},
\end{gathered}
$$

the CRS parabolic operator is given by

$$
\begin{gathered}
t\left(x_{m}, h ; \mathbf{m}\right)=t_{0}+\frac{2 \sin \alpha_{0}}{v_{0}}\left(x_{m}-x_{0}\right) \\
+\frac{2 t_{0} \cos ^{2} \alpha_{0}}{v_{0}}\left[\frac{\left(x_{m}-x_{0}\right)^{2}}{R_{\mathrm{N}}}+\frac{h^{2}}{R_{\mathrm{NIP}}}\right],
\end{gathered}
$$

and the hyperbolic form by

$$
t\left(x_{m}, h ; \mathbf{m}\right)=\sqrt{\begin{array}{c}
{\left[t_{0}+\frac{2 \sin \alpha_{0}\left(x_{m}-x_{0}\right)}{v_{0}}\right]^{2}+\frac{2 t_{0} \cos ^{2} \alpha_{0}}{v_{0}}} \\
\times\left[\frac{\left(x_{m}-x_{0}\right)^{2}}{R_{\mathbb{N}}}+\frac{h^{2}}{R_{\mathbb{N}}}\right]
\end{array}} .
$$

$R_{\mathrm{NIP}}=\frac{1}{K_{\mathrm{NI}}}, R_{\mathrm{N}}=\frac{1}{K_{\mathrm{N}}}$ and $\alpha_{0}$ are the wave front attributes, and the quantity $v_{0}$ stands for the velocity of the upper layer, that in practical work is established as a fixed value estimated around the stack reference point $P_{0}\left(x_{0}, t_{0}\right)$.

The justification for $v_{0}$ be included as an ambiguity parameter is that the values attributed to $v_{0}$ can vary, and can even be used as a filter for analyzing multiple attenuation. Besides, this velocity will depend on the source dominant band frequency, and velocity may considerably vary along the survey line, and even in its aperture; the exception would be marine surveys due to the smaller variation in water velocity.

Figure 2 simulates an observation surface (blue) calculated by ray theory, and the CRS stack surface (green) calculated by Eq. (4). The CRS stack has the intention to fit these two surfaces in terms of correlation Semblance analysis, and in the CRS case performed in an automatic way, and in steps summarized below. Each step searches for one of the attributes $\left(R_{\mathrm{NIP}}, R_{\mathrm{N}}, \alpha_{0}\right)$, and produces a stack related to each reference point $P_{0}\left(x_{0}, t_{0}\right)$, that are finally followed by 3-parameter optimization step. 


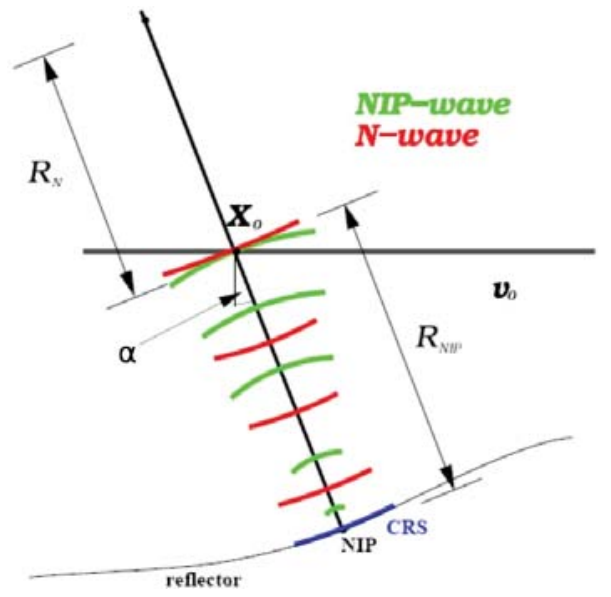

Figure 1 - Physical illustration of the CRS model formed by one layer over a half-space separated by a curved interface. The velocity $v_{0}$ characterizes the upper layer involving the observation aperture. The emergence angle $\alpha_{0}$ is common to both N and NIP waves. $R_{\mathrm{NIP}}$ is the NIP-wave radius and curvature $K_{\mathrm{NIP}}$, and $R_{\mathrm{N}}$ is the $\mathrm{N}$-wave radius and curvature $K_{\mathrm{N}}$ (Mann, 2002).

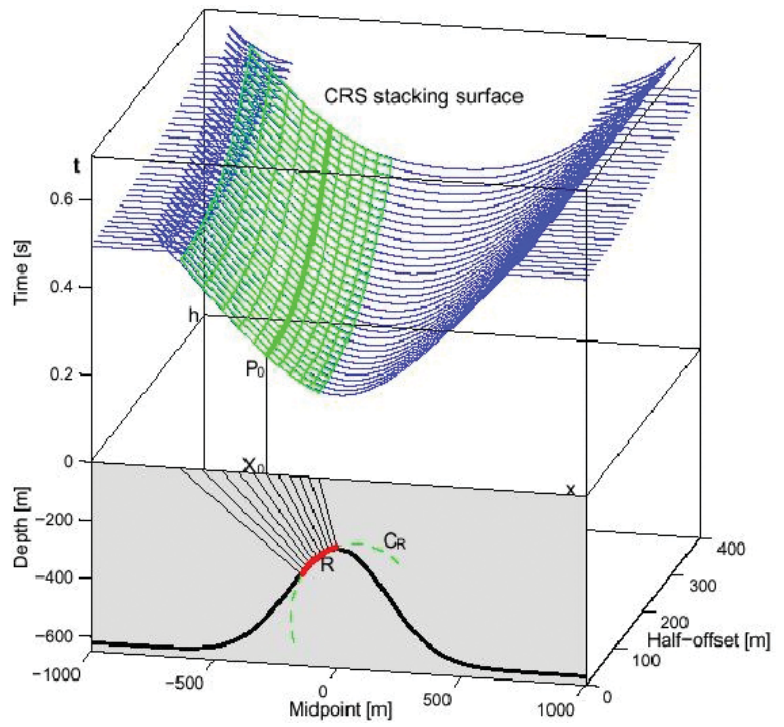

Figure 2 - 3D perspective of the surfaces to be indirectly fit in the stack process: the CRS operator in green [Eq. (4)], and the simulated observed data in blue (Mann, 2002).

To satisfy the Paraxial Ray Theory, the central ray of reference is taken the zero offset ray, or the Normal Incidence Ray in subsurface. This central ray satisfies Snell's law through the interfaces, and the wave front curvatures of the NIP and N waves change according to the refraction and transmission laws.

Fomel \& Kazinnik (2010) compares Multifocusing (MF) and CRS methods, where he proposes non-hyperbolic Common Reflection Surface approximations. Also, the CRS operator can be written in other forms for dealing with velocity analysis, like

$$
t\left(x_{m}, h ; \mathbf{m}\right)=t_{0}+A\left(x_{m}-x_{0}\right)+B\left(x_{m}-x_{0}\right)^{2}+C h^{2},
$$

where the three coefficients, $A, B$ and $C$, are related to the four parameters $v_{0}, R_{\mathrm{NIP}}, R_{\mathrm{N}}$ and $\alpha_{0}$, but the interest here are directly in the wave attributes (Duveneck, 2004).

Bernabini et al. (1987) describe functionals to be evaluated quantitatively on a given CMP gather for the goodness of fit between data and a model function, particularly for a stacking velocity value of the hyperbolic reflection response. The most common functional measures the likeness of the corrected gather's trace amplitudes $(\bar{u})$, based on correlation and choices of normalization. The extended normalized correlation Semblance measure, $\phi\left(t_{0} ; \mathbf{m}\right)$, to the $\left(h, x_{m}, t\right)$ coordinates, is composed by averages and given, without mathematical simplification, by:

$$
\begin{aligned}
& \phi\left(t_{0} ; \mathbf{m}\right)= \\
& \frac{\frac{1}{N_{t}} \sum_{t=t_{0}-\delta t}^{t=t_{0}+\delta t} \frac{1}{N_{x_{m}}} \sum_{x_{m}=x_{F}}^{x_{m}=x_{L}}\left[\frac{1}{N_{h}} \sum_{h=h_{F}}^{h=h_{L}} \bar{u}\left(x_{m}, h ; t\left(t_{0}\right), \mathbf{m}\right)\right]^{2}}{\sum_{t=t_{0}+\delta t} 1} \\
& \frac{1}{N_{t}} \sum_{t=t_{0}-\delta t}^{t=t_{0}+\delta t} \frac{1}{N_{x_{m}}} \sum_{x_{m}=x_{F}}^{x_{m}=x_{L}} \frac{1}{N_{h}} \sum_{h=h_{F}}^{h=h_{L}} \bar{u}^{2}\left(x_{m}, h ; t\left(t_{0}\right), \mathbf{m}\right)
\end{aligned}
$$

where the set of parameters $\mathbf{m}$ are related to the trajectory, $t=$ $t\left(t_{0}\right)$, of the summation defined by Eq. (4), and its different adapted forms. The $h$-window goes from a near first $h=h_{F}$ to a last $h=h_{L}$ offset with $N_{h}$ points. The $x_{m}$-window goes from a near first $x_{m}=x_{F}$ to a last $x_{m}=x_{L}$ mid-point with $N_{x}$ points. The $t$-window is specified by some $\delta t$ around $t_{0}$. $\phi\left(t_{0} ; \mathbf{m}\right)$ takes values in the interval $[0,1]$ regardless of the signal amplitude, and it quantifies the uniformity of the signal polarity across the normal moveout corrected gather amplitude, $\bar{u}(t)$.

Equation (6) is written in a form that does not explicitly carry information about the theoretical model, $t^{\text {(pre) }}\left(x_{m}, h\right)$, as Eq. (14) does; therefore, it is not used here as a direct curve fitting measure, and we call the stack process an indirect optimization process. Toldi (1985), and extensions referenced to his work (for example, Vieira et al. (2011)), treats the indirect optimization aiming at velocity estimation ( $v_{\mathrm{RMS}}$, or $v_{\text {INT }}$ ), with the trajectory $t=t\left(t_{0}\right)$ written in an explicit form of the type

$$
t\left(h ; t_{0, n}\right)=\sqrt{t_{0}^{2}+\frac{(2 h)^{2}}{v_{\mathrm{RMS}, n}^{2}}},
$$

where

$$
v_{\mathrm{RMS}, \mathrm{n}}^{2}=\frac{\sum_{i=1}^{n} v_{\mathrm{INT}, i}^{2} \Delta t_{i}}{\sum_{i=1}^{n} \Delta t_{i}},
$$

with time $\Delta t_{i}$ for vertical interval trajectories between two sequential reflections.

Figure 3 serves to represent the Semblance function, Eq. (6), where the aim of the optimization is to search for the global 


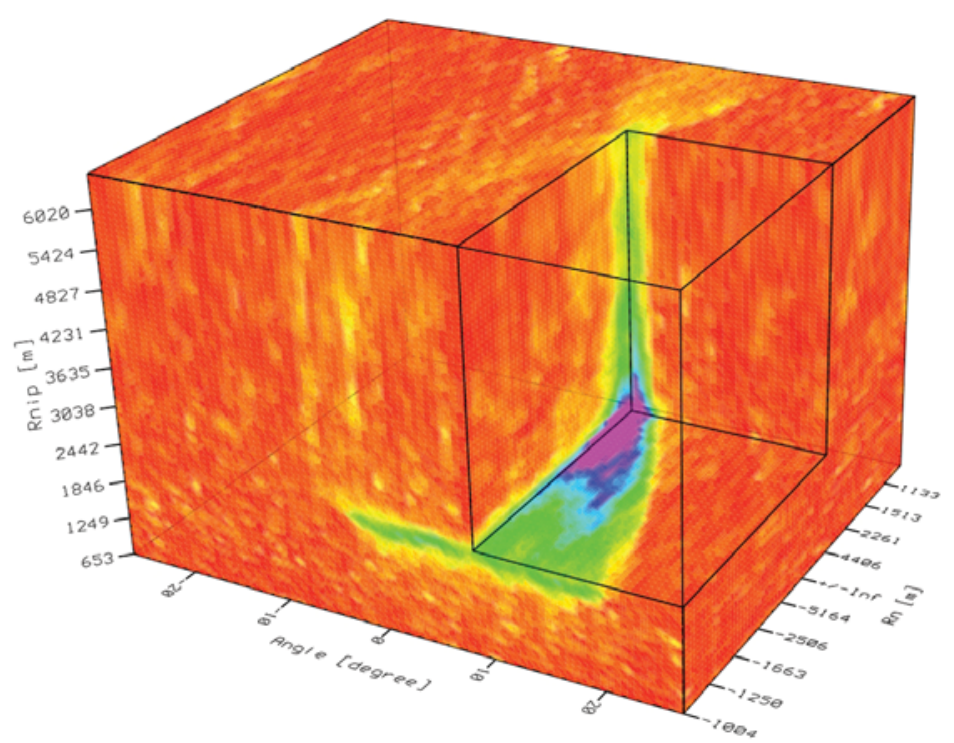

Figure 3 - Cube perspective of the coherence Semblance values calculated by Eq. (6) as function of the wavefield attributes $\alpha_{0}, R_{\mathrm{N}}$ and $R_{\mathrm{NIP}}$ (Mauch, 1999).

minimum represented in red. Local minimum can also be seen as yellow spots. Muller (1999) shows plots of Eq. (6) in form of cube perspectives and slices, as combination maps of $R_{\mathrm{NP}}$ versus $R_{\mathrm{N}}, R_{\mathrm{NIP}}$ versus $\alpha_{0}, R_{\mathrm{N}}$ versus $\alpha_{0}$, where the global minimum present a clearly elongated valley form.

The space sampling to construct the experiment were $\Delta x_{m}=50 \mathrm{~m}$ and $\Delta h=50 \mathrm{~m}$, with the respective number of points $N_{x_{m}}=50$ and $N_{h}=50$. The first geophones were placed at $h_{F}=50 \mathrm{~m}$ and $x_{F}=50 \mathrm{~m}$. The nominal values for the CRS parameters were $v_{0}=1500 \mathrm{~m} / \mathrm{s}, R_{\mathrm{NIP}}=+5000 \mathrm{~m}$, $R_{\mathrm{N}}=-5000 \mathrm{~m}, \alpha_{0}=0,2094 \mathrm{rad}$, and $t_{0}=1 \mathrm{~s}$.

Figure 4 represents the forward model (no noise added), and Figure 5 represents the synthetic data, obtained from Figure 4 where noise has been added to. Both informations were calculated by Eq. (4), and the additive random noise calculated with a normal distribution, and visually controlled. Therefore, this work does not have manually or automatic picked events, or real data.

\section{Attribute search}

Depending on the application, the number of the CRS attributes will be different, what also will depend on the dimension of the problem (if $2 \mathrm{D}$ or $3 \mathrm{D}$ ), and on the observation topography. For the flat observation surface and $2 \mathrm{D}$ problems, the model only considers the triplet $\left(R_{\mathbb{N I P}}, R_{\mathrm{N}}, \alpha_{0}\right)$. The parameter $v_{0}$ is admitted fixed, but the value adopted is considered as an a priori information, and to be decided for. We follow the descriptions of Muller (1999) and Mann (2002) for the stack implementation, where the triplet search is described as a nonlinear optimization problem; again, there is no search for $v_{0}$ in the practice of CRS stack.

For the triplet optimization step, it needs a starting point $\left(R_{\mathrm{NIP}}, R_{\mathrm{N}}, \alpha_{0}\right)^{(\text {(ni) }}$ that is obtained in previous specific steps performed in the CMP domain. The parameter picking should be associated with maximum coherence values used to simulate the correspondent ZO point, and finally the complete section.

First step. It is one-parameter search for the combined $v_{\text {stack }}$ for obtaining a first ZO section by setting $x_{m}=x_{0}$ in Eq. (4) that is reduced to:

$$
\left.t(h)\right|_{x_{m}=x_{0}}=\sqrt{t_{0}^{2}+\frac{2 t_{0} \cos ^{2} \alpha_{0}}{v_{0} R_{\mathrm{NIP}}} h^{2}} ;
$$

that, compared with

$$
t(h)=\sqrt{t_{0}^{2}+\frac{4 h^{2}}{v_{\mathrm{NMO}}^{2}}},
$$

the stacking velocity can be expressed in terms of $\alpha_{0}$ and $R_{\mathrm{NIP}}$, for $v_{\mathrm{NMO}}=v_{\text {stack }}$, as

$$
v_{\text {stack }}^{2}=\frac{2 v_{0} R_{\mathrm{NP}}}{t_{0} \cos ^{2} \alpha_{0}} .
$$

This step is called Automatic NMO stack (or, CMP stack), and it represents a non-interactive velocity analysis.

Second step. It is also one-parameter search for non-combined $\alpha_{0}$ attribute for obtaining a second ZO section by setting $h=0$ 


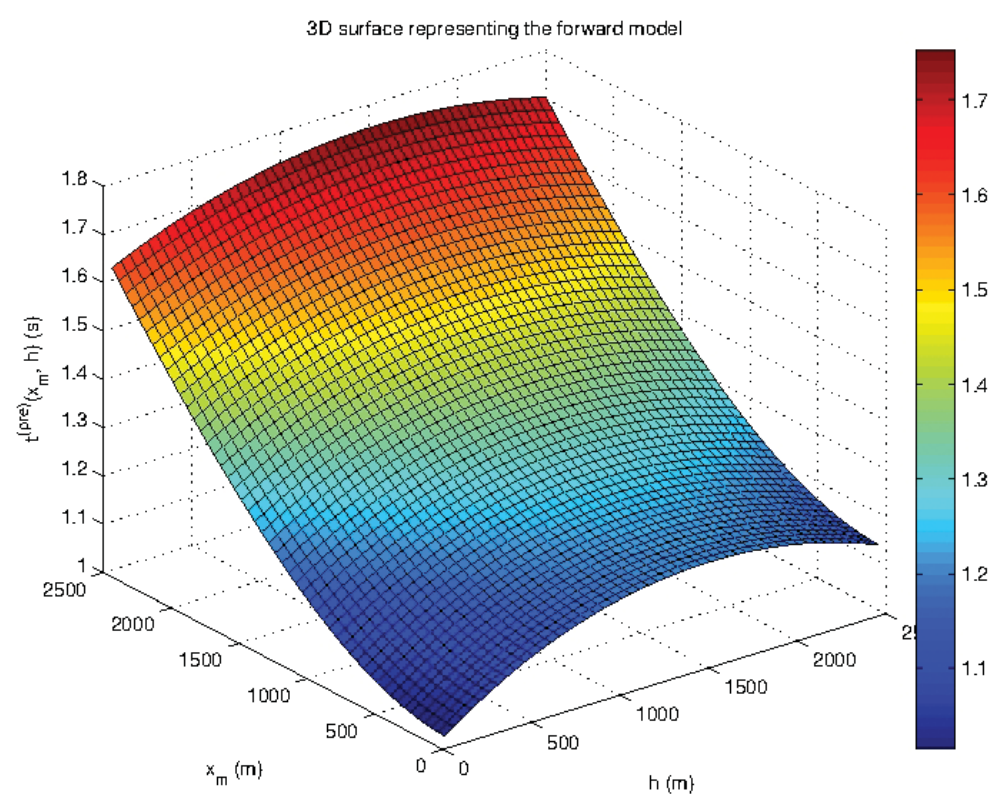

Figure 4-3D perspective of the time surface to be fit representing the forward model calculated by Eq. (4) showing the hyperbolic aspect along $h$ and $x_{m}$.

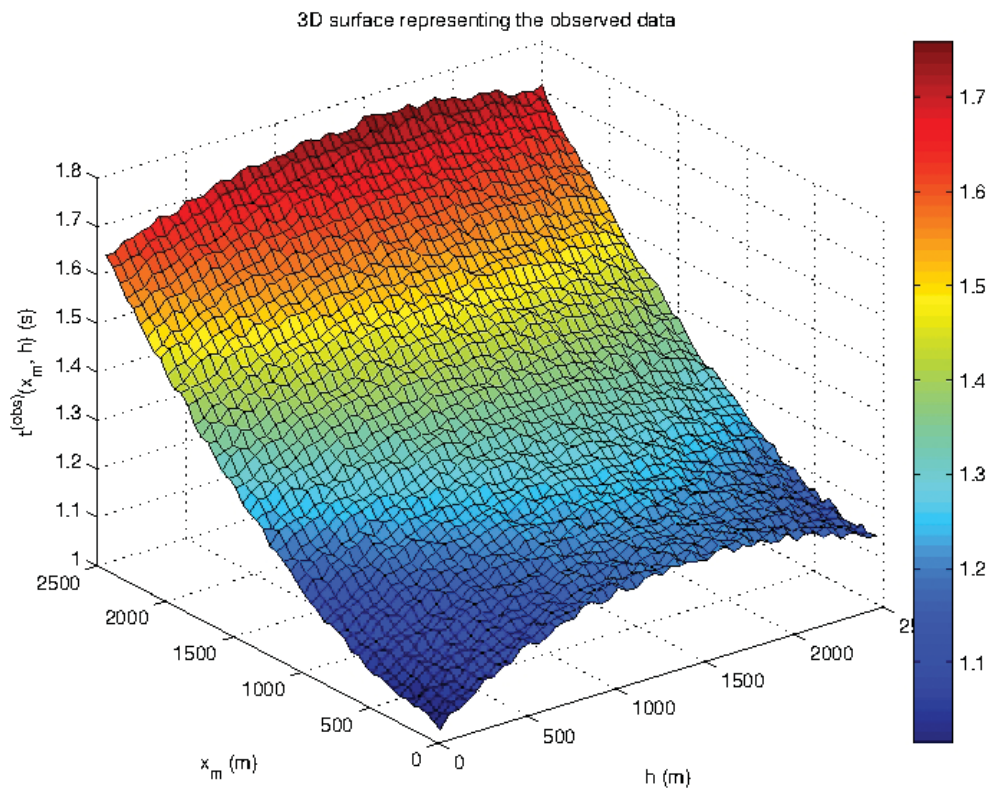

Figure 5 - 3D perspective of the time surface to be fit representing the synthetic data calculated by Eq. (4), where random noise was added to Figure 4, and still showing some hyperbolic aspect. The additive noise was calculated with $0.01 \%$ with respect to the maximum value of the traveltime.

and $R_{N}=\infty$ in Eq. (4) that reduces it to:

$$
\left.t\left(x_{m}\right)\right|_{\left(h=0, R_{N}=\infty\right)}=t_{0}+\frac{2 \sin \alpha_{0}\left(x_{m}-x_{0}\right)}{v_{0}},
$$

This first-order approximation can be regarded as a plane wave approximation, and this step is called Automatic Plane Wave stack, from where the emergence angle $\alpha_{0}$ is obtained based on a small aperture. Inserting this angle value into Eq. (8), a value for $R_{\mathrm{NIP}}$ is calculated.

Third step. It is one-parameter search for the non-combined $R_{\mathrm{N}}$ attribute for obtaining a third ZO section by setting $h=0$ 
in Eq. (4) that reduces it to:

$$
\left.t\left(x_{m}\right)\right|_{(h=0)}=\sqrt{\begin{array}{l}
{\left[t_{0}+\frac{2 \sin \alpha_{0}\left(x_{m}-x_{0}\right)}{v_{0}}\right]^{2}} \\
+\frac{2 t_{0} \cos ^{2} \alpha_{0}}{v_{0}} \frac{\left(x_{m}-x_{0}\right)^{2}}{R_{N}}
\end{array}} .
$$

In this step, the values of $\alpha_{0}$ and $R_{\text {NIP }}$ would already be known from the previous search, and this step is called Automatic Hyperbolic stack for $R_{\mathrm{N}}$.

Fourth step. From the previous 3 steps, the initial values $\left(R_{\mathrm{NIP}}, R_{\mathrm{N}}, \alpha_{0}\right)^{(\text {(ni) }}$ are known, and (with $v_{0}$ fixed) an Initial stack is performed to obtain a fourth ZO section.

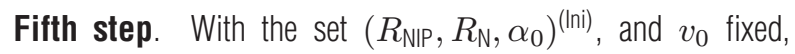
the simultaneous search provides the Optimized CRS stack producing another attribute set, $\left(R_{\mathrm{NIP}}, R_{\mathrm{N}}, \alpha_{0}\right)^{(0 \mathrm{pt})}$, and another ZO section.

Forward modeling can now be performed by calculating traveltime surfaces with $\mathrm{Eq}$. (4) and the set of attributes $\left(R_{\mathrm{NIP}}, R_{\mathrm{N}}, \alpha_{0}\right)^{(0 \mathrm{pt})}$. We can now take one randon subset for the sensitivity analysis.

\section{SENSITIVITY MODEL}

Sensitivity analysis can only be done on theoretical models, where closed derivatives can be taken, and by definition it is a model compromise. Therefore, sensitivity analysis on real data only makes partial sense here. Frank (1978) describes applications of model parameter sensitivity for physical prediction and applications. The system function is denoted by $t_{i}=t_{i}(\mathbf{m})$, dependent on the parameter vector $\mathbf{m}=\left[m_{1}, m_{2}, \ldots m_{M}\right]^{T}$, and in this case we have $\mathbf{m}$ specifically given by $\mathbf{m}=$ $\left[v_{0}, R_{\mathrm{NIP}}, R_{\mathrm{N}}, \alpha_{0}\right]^{T}$. The nominal parameters and system function are denoted with the lower script 0 in $\mathbf{m}_{0}$ and $t_{0}$.

Considering continuity conditions, the following definitions for the sensitivity function, matrix $\mathbf{S}$, are applied, and some are presented for comparison, and Appendix A is devoted to some details on this technique.

The adopted measure here was the Relative Logarithmic sensitivity function, $\overline{\mathbf{S}}$, defined by:

$$
\left.\bar{S}_{i j} \triangleq \frac{\partial \ln t_{i}(\mathbf{m})}{\partial \ln m_{j}}\right|_{m_{0}}=\bar{S}_{i j}\left(\mathbf{m}_{0}\right)=S_{i j} \frac{m_{0 j}}{t_{0 i}},
$$

where $S_{i j}$ is the $i j^{\text {th }}$ element of absolute sensitivity function $\mathbf{S}$ of Eq. (20). The $i^{\text {th }}$ element of the Relative Error of the system function is defined by:

$$
E_{\mathrm{R}}\left(x_{m}, h\right)=\frac{\Delta t_{i}}{t_{0 i}} \triangleq \sum_{j=1}^{j=M} S_{i j} \frac{\Delta m_{j}}{m_{0 j}},
$$

and the Maximum Relative Error of the system function by

$$
E_{\mathrm{A}}\left(x_{m}, h\right)=\left|\frac{\Delta t_{i}}{t_{0 i}}\right| \triangleq \sum_{j=1}^{j=M}\left|S_{i j}\right|\left|\frac{\Delta m_{j}}{m_{0 j}}\right| .
$$

Examples of the $\overline{\mathbf{S}}$ function are presented in Figures 10, 11, 12 and 13 .

\section{OPTIMIZATION METHODS}

This short description is to present a relation between the optimization technique and the sensitivity analysis for completeness. Vieira \& Leite (2009) presented strategies with two optimization methods combined to investigate the convergence and resolution of the CRS operator aiming at practical applications. The concepts involved are the Random Controlled Search, and the Gradient methods. The misfit measure used, $\chi(\mathbf{m})$, is given by:

$$
\chi(\mathbf{m})=\sqrt{\frac{\sum_{i=1}^{N}\left[t_{i}^{(\mathrm{obs})}-t_{i}^{(\mathrm{pre})}(\mathbf{m})\right]^{2}}{N} .}
$$

This measure is not to be confused with the Semblance function, and Figure 6 shows the layout of the inversion process. The function $\chi(\mathbf{m})$ has the properties to be positive, convex, multimodal, and not necessarily smooth, what will depend on the $\Delta \mathbf{t}(\mathbf{m})=\mathbf{t}^{\mathrm{obs}}-\mathbf{t}^{\mathrm{pre}}(\mathbf{m})$ relation. The parameter, $\mathbf{m}$, search is to minimize the function $\chi(\mathbf{m})$.

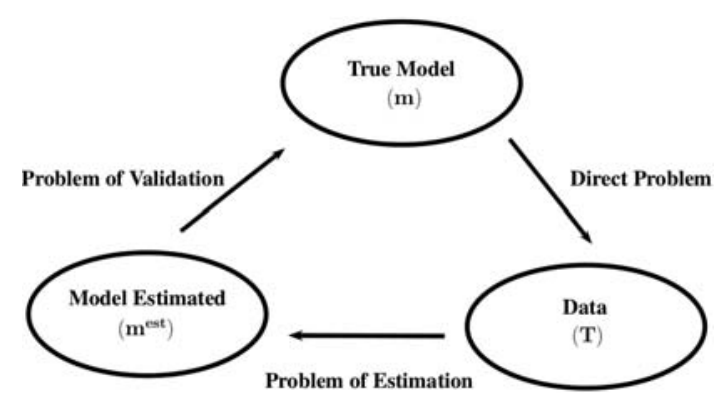

Figure 6 - Canonic representation of the optimization principle.

The CRSGM formalism applied was originally described by Price (1983) to solve the global optimization problem, and Appendix $B$ is devoted to some details on this method to show the necessity of control, what makes the curve fitting process a little more nonlinear. A common characteristic of the global methods, as described by Brachetti et al. (1997), is that they attack two distinct problems at the same time: 
1. The global search problem that is the examination of all region of interest aiming at to localize "more promising" sub-regions that contains the global minimum $\left(\mathbf{m}^{* *}\right)$;

2. The local search problem that is the determination of the global minimum $\left(\mathbf{m}^{* *}\right)$ using a local strategy, once a rather small neighborhood has been detected around the minimum.

The Gradient Method (GM) is a local search formalism described to some details in Appendix C. The main point is that the GM is based on the multivariate Taylor series expansion of a function used to represent the observed data in a nonlinear problem, that is summarized as $\mathbf{G}(\mathbf{m}) \Delta \mathbf{m}=\Delta \mathbf{t}$, where $\mathbf{G}(\mathbf{m})$ is the problem matrix function of the parameters $\mathbf{m}$ to be resolved for iteratively. The series linearizes the problem to allow for an iterative solution, which is based on the linear counterpart form, $\mathbf{G m}=\mathbf{t}$, where $\mathbf{G}$ is not a function of the $\mathbf{m}$ parameters.

In the least-square sense, the optimization here is defined as an over-determined (pure) problem, the number of data being greater then the number of parameters to be resolved for, $(N>M)$, and all parameters considered to have the same illumination. The minimization method uses the principle that $\partial \chi(\mathbf{m}) / \partial m_{j}=0$, what establishes a local minimum, with the solution represented by the normal equation $\mathbf{G}(\mathbf{m}) \Delta \mathbf{m}=\Delta \mathbf{t}$. The iterative solution of the nonlinear problem is represented by the equation:

$$
\left.\Delta \mathbf{m}\right|_{k}=\left.\left[\mathbf{G}^{T} \mathbf{G}\right]^{-1} \mathbf{G}^{T} \Delta \mathbf{t}\right|_{k},
$$

under the parameter update given by

$$
\mathbf{m}^{(k+1)}=\mathbf{m}^{(k)}+\gamma^{(k)} \Delta \mathbf{m}^{(k)},
$$

where $\gamma$ is an attenuation/amplification factor for the solution $\Delta \mathbf{m}$, and $k$ is the iteration number in the optimization process cycle.

A statistical analysis for the method is done by the Data and Parameter Resolution Matrices, and by the Unitary Covariance Matrix (Menke, 2002). These matrices are attributes of the method involving the matrix $\mathbf{G}$, and its generalized inverse $\mathbf{G}^{-g}$, that in the present case has the form

$$
\mathbf{G}^{-g}=\left[\mathbf{G}^{T} \mathbf{G}\right]^{-1} \mathbf{G}^{T} .
$$

The Parameter Resolution matrix, $\mathbf{R}_{p}$, is given by:

$$
\mathbf{R}_{p}=\mathbf{G}^{-g} \mathbf{G},
$$

the Data Resolution matrix, $\mathbf{R}_{d}$, by

$$
\mathbf{R}_{\mathrm{d}}=\mathbf{G G}^{-g},
$$

and the Unitary Covariance matrix, $\mathbf{c o v}_{u}$, by

$$
\operatorname{cov}_{u}(\mathbf{m})=\sigma^{-2} \mathbf{G}^{-g}[\operatorname{cov}(\mathbf{t})] \mathbf{G}^{-g T}=\mathbf{G}^{-g} \mathbf{G}^{-g T} .
$$

The CRSGM is a classified as a Direct Search Method, and therefore does not have statistical operators as for the GM described above.

Examples of the two optimization processes are presented below in the Results section, where control values for the inversion processes are also given immediately after the calculation flow description.

\section{RESULTS}

The calculation flow description of the computer program Vieira \& Leite (2009) developed by is resumed as follows:

01 - Start;

02 - Input: (1) Control parameters; (2) Model parameters; (3) Initial model parameters; (4) Inversion parameters;

03 - Start iterations: Controlled Random Search Method;

04 - End iterations: Controlled Random Search Method;

05 - Start iterations: Gradient Method;

06 - Calculation: Forward model;

$\mathbf{0 7}$ - Calculation: Curve fit measure, and test for ending the iterations;

08 - Calculation: Derivatives of the CRS stack operator;

$\mathbf{0 9}$ - Calculation: Matrices $\mathbf{G}, \Delta \mathbf{d}, \Delta \mathbf{m}$;

10 - Calculation: Parameter update;

11 - Calculation: Convergence test;

12 - Returns to 05;

13 - Calculation: Parameter Resolution matrix;

14 - Calculation: Data Resolution matrix;

15 - Calculation: Unit Covariance Unitary matrix.

The Price initial interval control parameters were set in the computer program to the following values. For $v_{0}$ : 1400 $1600 \mathrm{~m} / \mathrm{s}$, with the center value of $1500 \mathrm{~m} / \mathrm{s} ; R_{\mathrm{NIP}}: 4500$ to $5500 \mathrm{~m}$, with the center value of $5000 \mathrm{~m} ; R_{\mathrm{N}}:-5500$ to $-4000 \mathrm{~m}$, with center value of $-5000 \mathrm{~m}$; and $\alpha_{0}: \pi / 18$ to $\pi / 12 \mathrm{rd}$, with center value of $\pi / 15 \mathrm{rd}$. The parameter space has dimension 4 , and the number of points in the parameter space were set to 200 , with a tolerance of $0.01 \%$. Figure 7 serves to exemplify the 


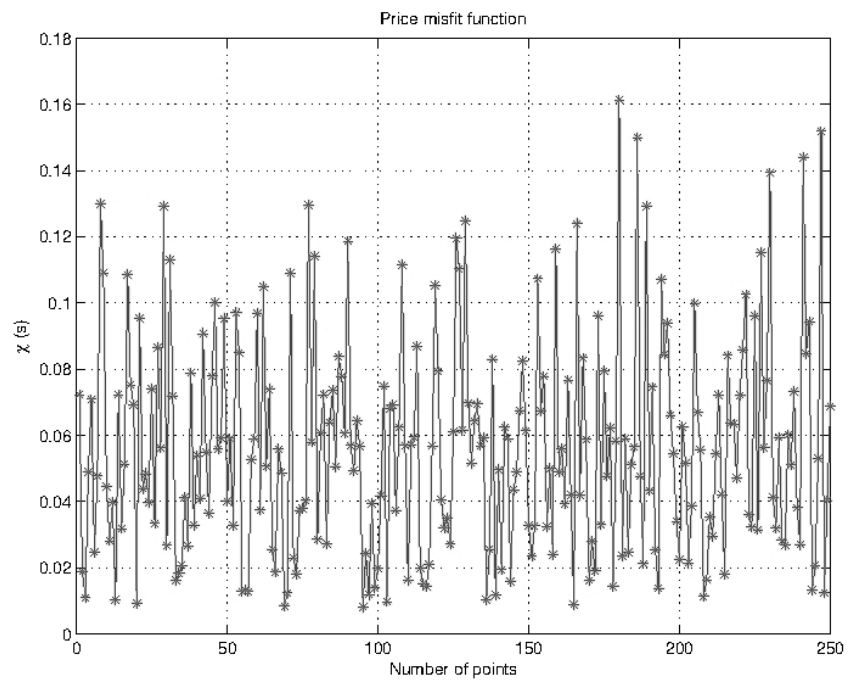

Figure 7 - Price initial random misfit function.

randomness of the misfit function $\chi(\mathbf{m})$ as a result of the automatic random selection of $\mathbf{m}$ for $t_{0}=1.0 \mathrm{~s}$.

The GM inversion process were set to 20 iterations. The GM control parameters had the following initial values: $v_{0}=$ $1800 \mathrm{~m} / \mathrm{s} ; R_{\mathrm{NIP}}=5700 \mathrm{~m} ; R_{\mathrm{N}}=-4200 \mathrm{~m} ; \alpha_{0}=\pi / 10 \mathrm{rd}$; the regularization factor $\gamma=1.0 \mathrm{e}-7$; and $t_{0}=1.0 \mathrm{~s}$.

Table 1 presents the values obtained by the application of the CRSGM and GM methods, with the above control parameters, which shows agreement between the results, and to be a strong allied in the strategy for the 4 parameters search of the CRS forward model. We should make clear that the accuracy obtained depends on the inversion control parameters, and on the number of iterations. Since we are dealing with two independent methods, we intentionally chose for the independent solutions and, therefore, the values for CRSGM and GM were left close to the nominal values.

Table 1 - Inversion values obtained by the CRSGM and GM optimization methods.

\begin{tabular}{|c|c|c|c|}
\hline Parameter & Real & CRSGM & GM \\
\hline$v_{0}(\mathrm{~m} / \mathrm{s})$ & 1500 & 1459 & 1446 \\
\hline$R_{\mathrm{NIP}}(\mathrm{m})$ & 5000 & 5013 & 5112 \\
\hline$R_{\mathrm{N}}(\mathrm{m})$ & -5000 & -4910 & -4800 \\
\hline$\alpha_{0}$ (radians) & 0.2094 & 0.2019 & 0.2186 \\
\hline
\end{tabular}

Table 2 presents the Normalized Parameter Resolution $(4 \times 4)$ matrix of Eq. (17) that, should ideally have a unitary diagonal form, and shows a weak scatter in the off diagonal elements. The correlation values in the $\alpha_{0}$ columns are very small, (smaller than
0.0001), with respect to the other parameters, and they serve as a measure of relative parameter independence.

Table 2 - Normalized Parameter Resolution matrix limited to 3 decimal places. Eq. (17).

\begin{tabular}{|c|c|c|c|c|}
\hline $\mathbf{R}_{p}$ & $v_{0}$ & $R_{\mathrm{NP}}$ & $R_{\mathrm{N}}$ & $\alpha_{0}$ \\
\hline$v_{0}$ & 1 & 0.287 & -0.173 & 0.000 \\
\hline$R_{\mathrm{NIP}}$ & 0.287 & 1 & 0.893 & 0.000 \\
\hline$R_{\mathrm{N}}$ & -0.173 & 0.893 & 1 & 0.000 \\
\hline$\alpha_{0}$ & 0.000 & 0.000 & 0.000 & 1 \\
\hline
\end{tabular}

Figure 8 shows the Normalized Decimated Data Resolution $(50 \times 50)$ matrix of Eq. (18). This matrix had originally dimensions $(2500 \times 2500)$, but was decimated for better plotting purpose, but conserved its original form and scale. This representation with contours is more convenient than as a table. This matrix should ideally have a unitary diagonal form, but shows a strong scatter of the off diagonal elements.

Table 3 - Normalized Unitary Parameter Covariance matrix limited to 3 decimal places. Eq. (19).

\begin{tabular}{|c|c|c|c|c|}
\hline $\operatorname{COV}_{u}(\mathbf{m})$ & $v_{0}$ & $R_{\mathrm{NIP}}$ & $R_{\mathrm{N}}$ & $\alpha_{0}$ \\
\hline$v_{0}$ & 1 & -0.832 & -0.855 & -0.362 \\
\hline$R_{\mathrm{NIP}}$ & -0.832 & 1 & 0.999 & 0.772 \\
\hline$R_{\mathrm{N}}$ & -0.855 & 0.999 & 1 & 0.750 \\
\hline$\alpha_{0}$ & -0.362 & 0.772 & 0.750 & 1.0 \\
\hline
\end{tabular}

Table 3 presents the Normalized Unitary Parameter Covariance $(4 \times 4)$ matrix of Eq. (19), that should ideally have a unitary diagonal form, but shows a strong scatter of the off diagonal 


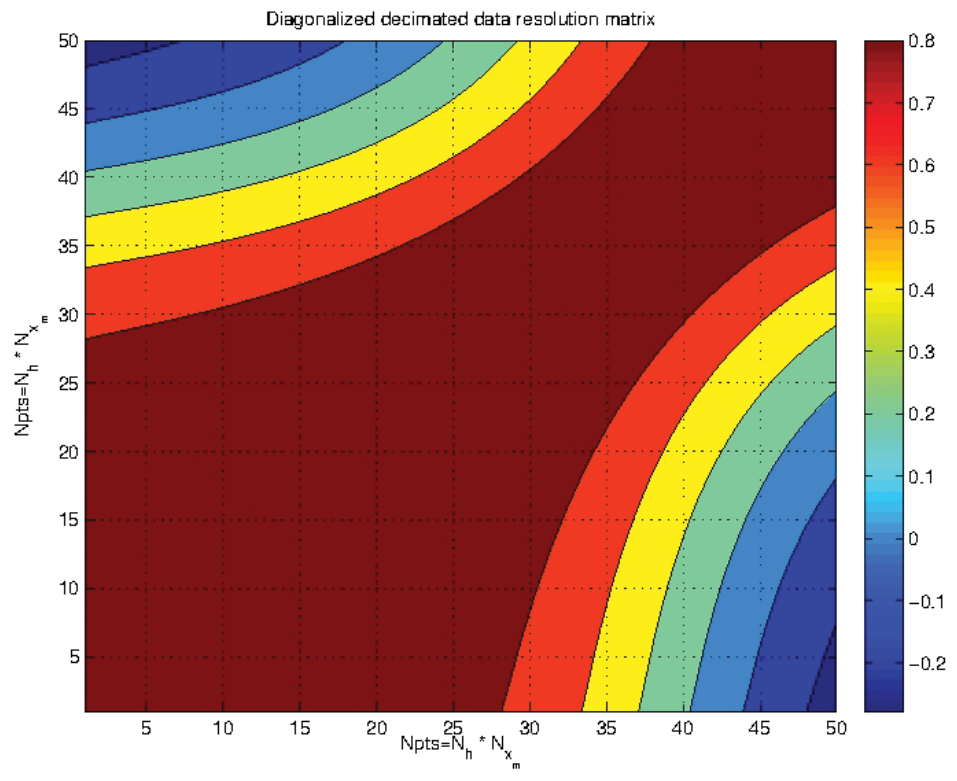

Figure 8 - Data resolution matrix decimated by 50 points.

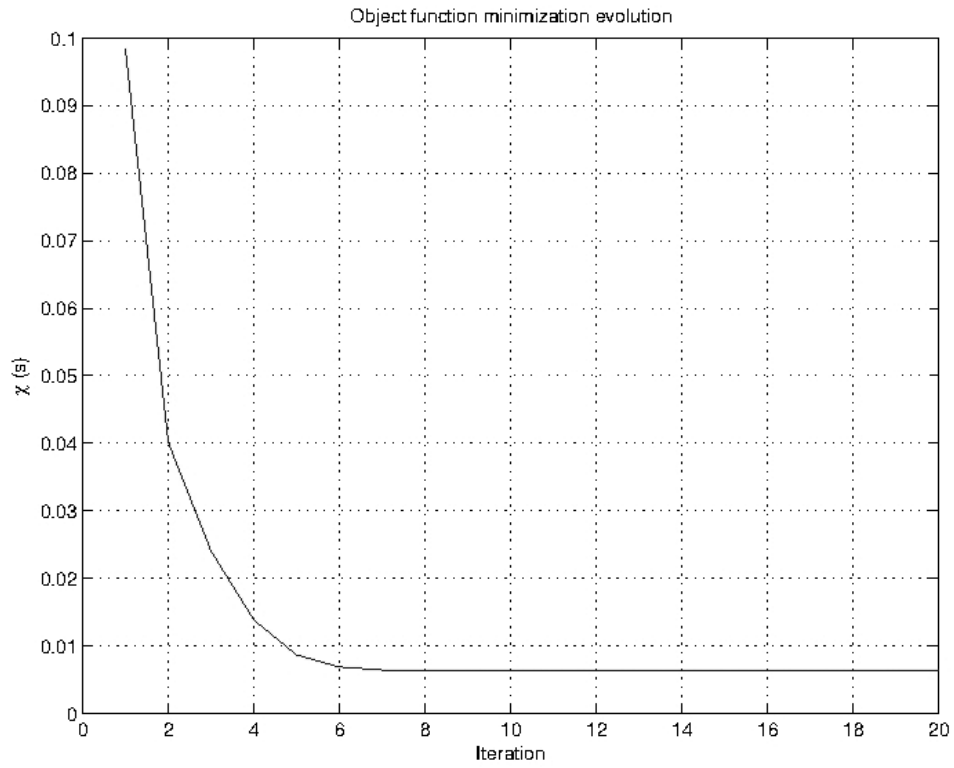

Figure 9 - Evolution of the objective minimization function calculated in 20 iterations showing the stabilization of the process before the tenth iteration.

elements. This representation in form of table is more convenient than as a figure with contours. The values shown correspond to a non-desirable strong correlation between the parameters, and means that by changing one parameter the others are also altered according to the sign as, for example, between $R_{\mathrm{N}}$ and $R_{\mathrm{NIP}}$ with $\operatorname{cov}_{u}=0.999$.

The maximum number of iterations in the GM method was limited to 20, and the evolution of the minimization object func- tion is shown in Figure 9. It was established that the solution by the CRSGM was to be used as input to the GM method. But, for the above tables, they were obtained in independent experiments, to check for the convergence of the methods. Also, the Global method is only used to give the initial point in the parameter space for calculating the statistics based on the local method. The experiment results show that a precise local point is not a decisive matter due to the linearity at the local minimum. 

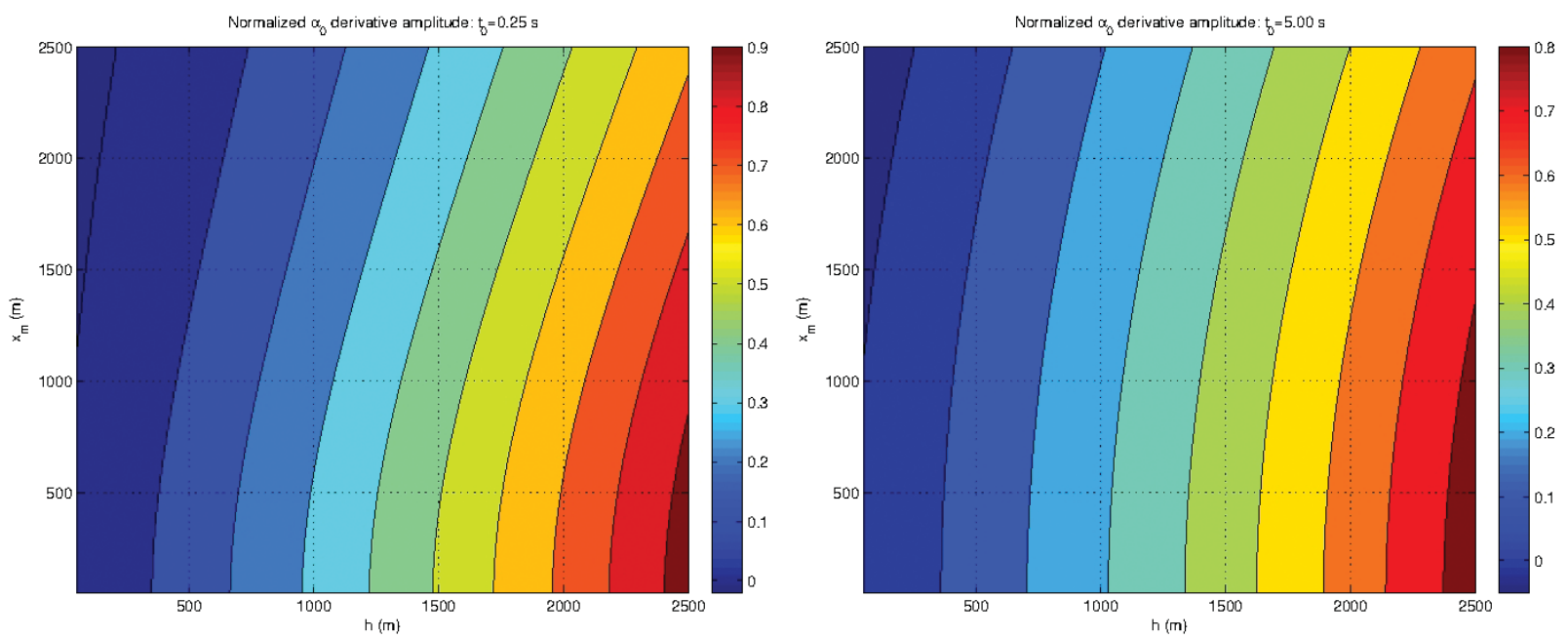

Figure 10 - Contour maps of the normalized derivative $\left(\alpha_{0} / t_{0}\right) * \partial t\left(x_{m}, h ; \mathbf{m}\right) / \partial \alpha_{0}$. Figure positions: left, $t_{0}=0.25 \mathrm{~s} ;$ right, $t_{0}=5.00 \mathrm{~s}$.
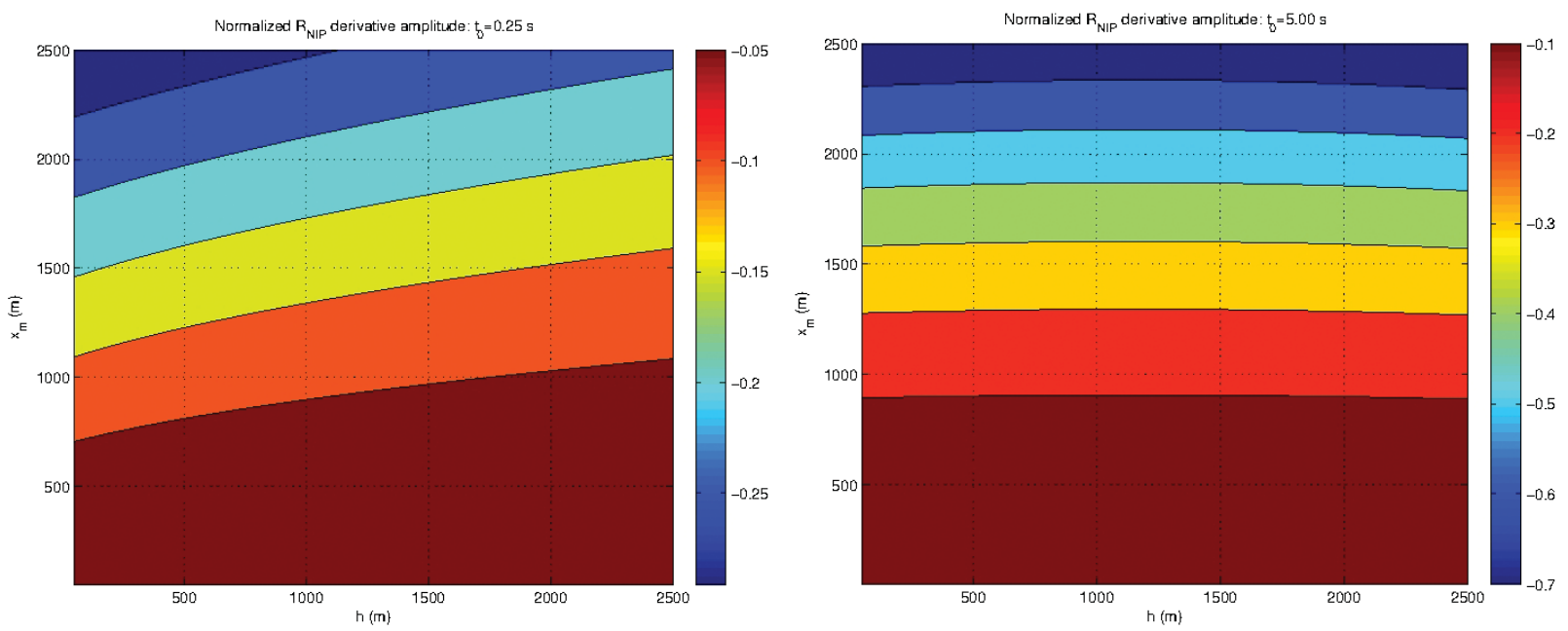

Figure 11 - Contour maps of the normalized derivative $\left(R_{\mathrm{NIP}} / t_{0}\right) * \partial t\left(x_{m}, h ; \mathbf{m}\right) / \partial R_{\mathrm{NIP}}$. Figure positions: left, $t_{0}=0.25 \mathrm{~s}$; right, $t_{0}=5.00 \mathrm{~s}$.

The possible number of experiments with the sensitivity function $\overline{\mathbf{S}}$, Eq. (23), is theoretically equal to the number of points in a ZO section (time versus distance); therefore, it can be very large, and criteria must be used to select points for analysis. Examples are presented in Figures 10, 11, 12 and 13, where the nominal values were

$$
\begin{gathered}
v_{0}=1500 \mathrm{~m} / \mathrm{s}, \quad R_{\mathrm{NP}}=5000 \mathrm{~m}, R_{\mathrm{N}}=-5000 \mathrm{~m}, \\
\text { and } \quad \alpha_{0}=+(\pi / 15) \mathrm{rad} .
\end{gathered}
$$

The general chosen time points were $t_{0 i}=(0.25,0.50,1.00$, $2.00,2.50,3.00,4.00,5.00) \mathrm{s}$, with selected values for each case.

Figures 10,11 and 12 were calculated for only two values of $t_{0 i}=(0.25,5.00) \mathrm{s}$, necessary to show because the function variation is very smooth.
Figure 10 is the simplest case, and involves the function $\left(\alpha_{0} / t_{0}\right) * \partial t\left(x_{m}, h ; \mathbf{m}\right) / \partial \alpha_{0}$, Eq. (42), and its relation to Eq. (9). The maps show a strong linear dependence with respect to the coordinates $h$, and a weak linear dependence with respect to the $x_{m}$ coordinate; as a result, this parameter would be better determined along the $x_{m}$ coordinate constrained to a $h$ value. In the attribute search strategies, the second step solves for $\alpha_{0}$, as expressed by Eq. (9) for $t=t\left(x_{m}, h=0\right)$.

Figure 11 shows maps of the derivative $\left(R_{\mathrm{NP}} / t_{0}\right) * \partial t\left(x_{m}\right.$, $h ; \mathbf{m}) / \partial R_{\mathrm{NIP}}$, Eq. (43), and its relation to Eq. (7). The maps show a linear dependence with respect to $x_{m}$, and almost constant with respect to $h$, what makes this parameter less sensitive and stable along the $h$ axis; as a result, this parameter would be better determined along the $h$ coordinate constrained to a $x_{m}$ 

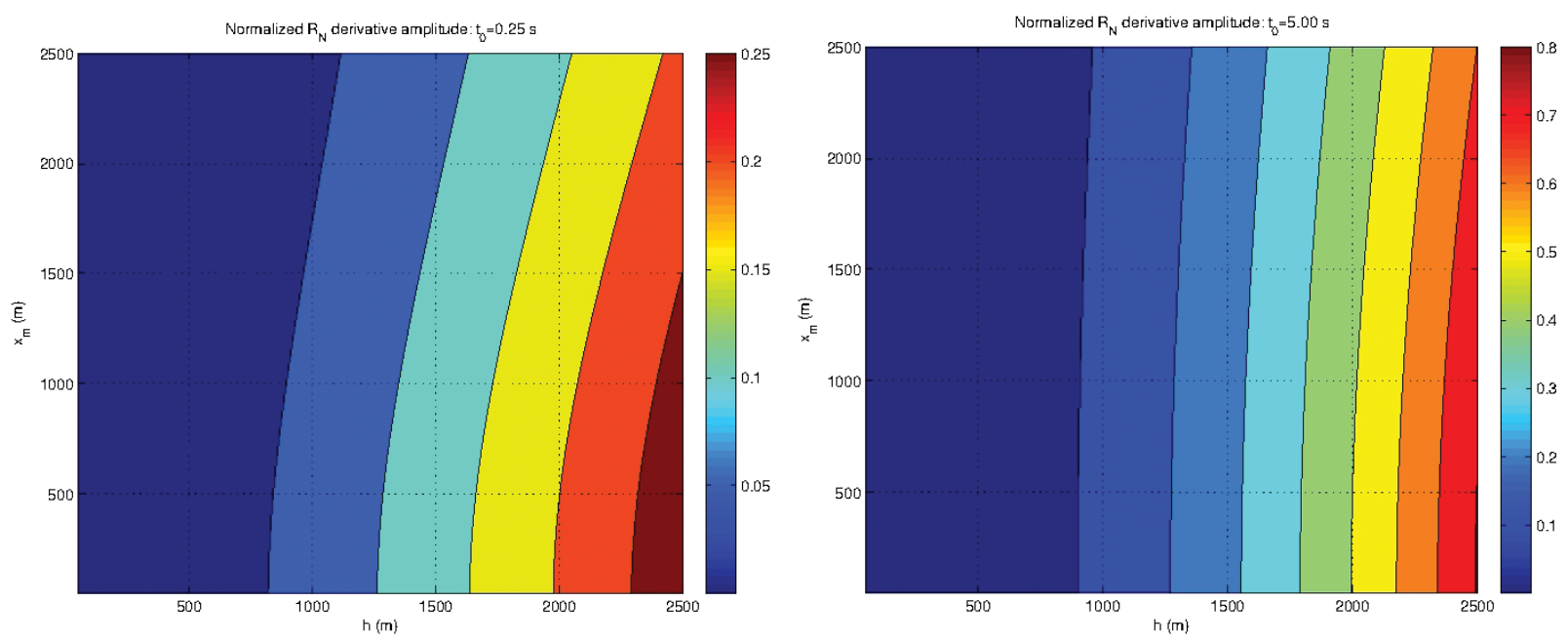

Figure 12 - Contour maps of the normalized derivative $\left(R_{\mathrm{N}} / t_{0}\right) * \partial t\left(x_{m}, h ; \mathbf{m}\right) / \partial R_{\mathrm{N}}$. Figure positions: left, $t_{0}=0.25 \mathrm{~s}$; right, $t_{0}=5.00 \mathrm{~s}$.

value. In the attribute search strategies, a combination of the first and second steps solves for $R_{\mathrm{NIP}}$, as expressed by Eq. (7) for $t=t\left(h, x_{m}=0\right)$.

Figure 12 shows maps of the derivative $\left(R_{\mathrm{N}} / t_{0}\right) * \partial t\left(x_{m}\right.$, $h ; \mathbf{m}) / \partial R_{\mathrm{N}}$, Eq. (44), and its relation to Eq. (10). The maps show a strong linear dependence with respect to the $h$ coordinate, and almost constant with respect to the $x_{m}$ coordinate; as a result, this parameter would be better determined along the $x_{m}$ coordinate constrained to a $h$ value. In the attribute search strategies, the third step solves for $R_{N}$, as expressed by Eq. (10) for $t=t\left(x_{m}, h=0\right)$. This situation is opposite to the $\left(R_{\mathrm{NIP}} / t_{0}\right) * \partial t\left(x_{m}, h ; \mathbf{m}\right) / \partial R_{\mathrm{NIP}}$ case.

Figure 13 displays the derivative $\left(v_{0} / t_{0}\right) * \partial t\left(x_{m}, h ; \mathbf{m}\right) /$ $\partial v_{0}$, Eq. (45), and its relation to the constant value of $v_{0}$. The variation is still smooth, but faster than for the other three parameters $\left(R_{\mathrm{NIP}}, R_{\mathrm{N}}\right.$ and $\left.\alpha_{0}\right)$. The maps indicate a nonlinear variation with respect to the space coordinates $\left(x_{m}\right.$ and $\left.h\right)$, without an ideal direction for $v_{0}$ initial evaluation with respect to the nominal value of $v_{0}=1500 \mathrm{~m} / \mathrm{s}$ positioned at the center of the figures. The maps indicate a very slow change with respect to higher $t_{0 i}$ values, and to be more complex for smaller $t_{0 i}$ values. Therefore, fixing the value of $v_{0}$ in the CRS practice is a consistent constraint for the implementation and simplification of the data stack processing algorithm, with respect to the $\mathbf{S}$ analysis.

Figure 14 are error functions calculated using Eqs. (12) and (13) of the adopted sensitivity measure, $\overline{\mathbf{S}}$. The maps show clearly and consistently a behavior of decreasing/increasing error with coordinates $h$ and $x_{m}$, but mainly along the $h$ axis, as a property of the hyperbolic operator (4). The elongated valleys of low values, along $h=1500 \mathrm{~m}$, coincide with the nominal values in Eqs. (12) and (13), that give minimum values for $\Delta t_{i}$.

\section{CONCLUSIONS}

We investigated the relationship between sensitivity functions for the CRS operator with respect to the parameters $\left(v_{0}, R_{\mathrm{NIP}}, R_{\mathrm{N}}\right.$ and $\left.\alpha_{0}\right)$, and adopted the logarithm measure $\overline{\mathbf{S}}$. Then, we compared the $\overline{\mathbf{S}}$ results with the attributes search strategies for $\left(R_{\mathrm{NPP}}\right.$, $R_{\mathrm{N}}$ and $\alpha_{0}$ ) that are based on physical-mathematical models for the construction of the CRS stack operator.

In the CRS stack, $v_{0}$ is admitted as fixed, but it has a physical meaning as shown in Figure 2. In practical terms, $v_{0}$ is represented by an average value taken along the geophone spread, and a sampling over the upper layers under the dominant wavelength window of the effective source pulse. The strategy for fixed $v_{0}$ is consistent with the $\overline{\mathbf{S}}$ analysis, due to the stronger variation of the $v_{0}$ sensitivity function with respect to the space coordinates, as presented in Figure 13, where the main attention was called for.

The $\mathbf{S}$ derivatives show some nonlinear behavior with respect to the spatial coordinates, $\left(x_{m}, h\right)$, but complementary strategies for the parameter search were not here provided to establish the initial set of values $\left(R_{\mathrm{NIP}}, R_{\mathrm{N}}, \alpha_{0}\right)^{\text {(Ini) }}$ to be used in the simultaneous optimization step for obtaining $\left(R_{\mathrm{NIP}}, R_{\mathrm{N}}, \alpha_{0}\right)^{\text {(0pt) }}$.

For the parameter resolution, we have systematically concluded in the text that it is necessary a good start point for the optimization that searches simultaneously for the three parameters $\left(R_{\mathrm{NIP}}, R_{\mathrm{N}}, \alpha_{0}\right)^{\text {(opt) }}$ with $v_{0}$ fixed. From the interpretation of the sensitivity functions, it is necessary to employ constraints as a priori conditions for the simultaneous parameter search.

The tests presented were performed for a fixed point 

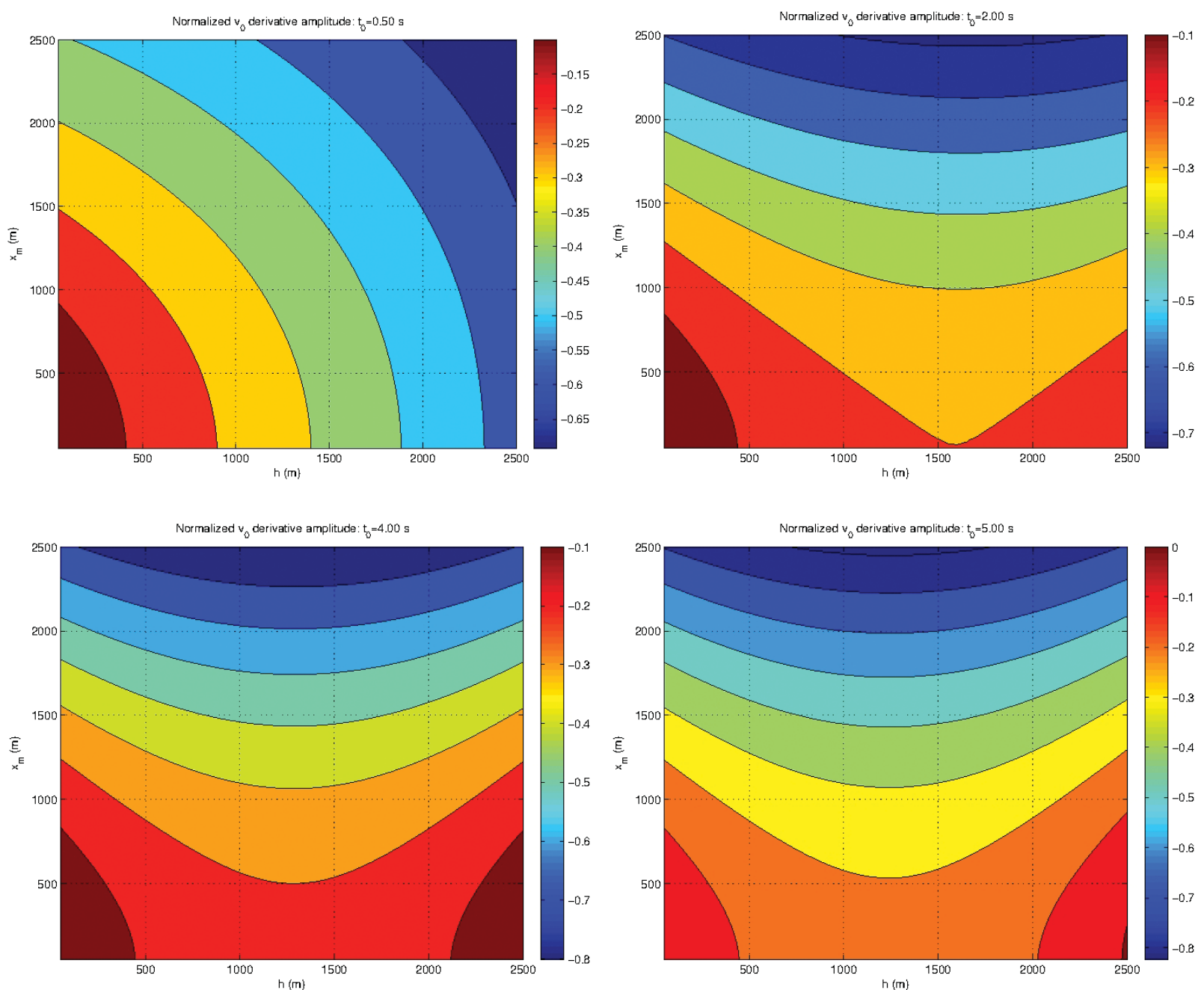

Figure 13 - Contour maps of the normalized derivative $\left(v_{0} / t_{0}\right) * \partial t\left(x_{m}, h ; \mathbf{m}\right) / \partial v_{0}$ for the nominal values $v_{0}=1500 \mathrm{~m} / \mathrm{s}$. Figure positions: top left, $t_{0}=0.50 \mathrm{~s}$; top right, $t_{0}=2.00 \mathrm{~s}$; bottom left, $t_{0}=4.00 \mathrm{~s}$; bottom right, $t_{0}=5.00 \mathrm{~s}$. From the experiments, the form varies very little after $t_{0}=2 \mathrm{~s}$.

$P_{0}\left(x_{0}, t_{0}\right)$. Another strategy would test for randomly chosen points along a trace, $\left(t_{0}=0, t_{\text {total }}\right)$, and for any CMP.

Once established a form to represent the ambiguity and sensitivity of the $t\left(x_{m}, h ; \mathbf{m}\right)$ function to its parameters, a next step would also be to repeat the experiment based on a form of modified fitting norm function.

\section{Appendix A: The Sensitivity Functions}

This description follows mostly Frank (1978) and Saltelli et al. (2004), where applications for different scientific fields are presented.

The Absolute sensitivity function is defined $(\triangleq)$ by:

$$
\left.S_{i j} \triangleq \frac{\partial t_{i}(\mathbf{m})}{\partial m_{j}}\right|_{\mathbf{m}_{0}}=\begin{aligned}
& S_{i j}\left(\mathbf{m}_{0}\right),(i=1,2, \ldots, N), \\
& (j=1,2, \ldots, M),
\end{aligned}
$$

the parameter-induced error by

$$
\Delta t_{i} \triangleq \sum_{j=1}^{M} S_{i j} \Delta m_{j}
$$

and the maximum error by

$$
\left|\Delta t_{i}\right| \triangleq \sum_{j=1}^{M}\left|S_{i j}\right|\left|\Delta m_{j}\right|,
$$

where the vertical bars stand for the absolute values of the elements of the corresponding vector $\mathbf{m}$ or $S_{i j}$.

The Relative Logarithmic sensitivity function, $\overline{\mathbf{S}}$, is defined by:

$$
\left.\bar{S}_{i j} \triangleq \frac{\partial \ln t_{i}(\mathbf{m})}{\partial \ln m_{j}}\right|_{m_{0}}=\bar{S}_{i j}\left(\mathbf{m}_{0}\right)
$$



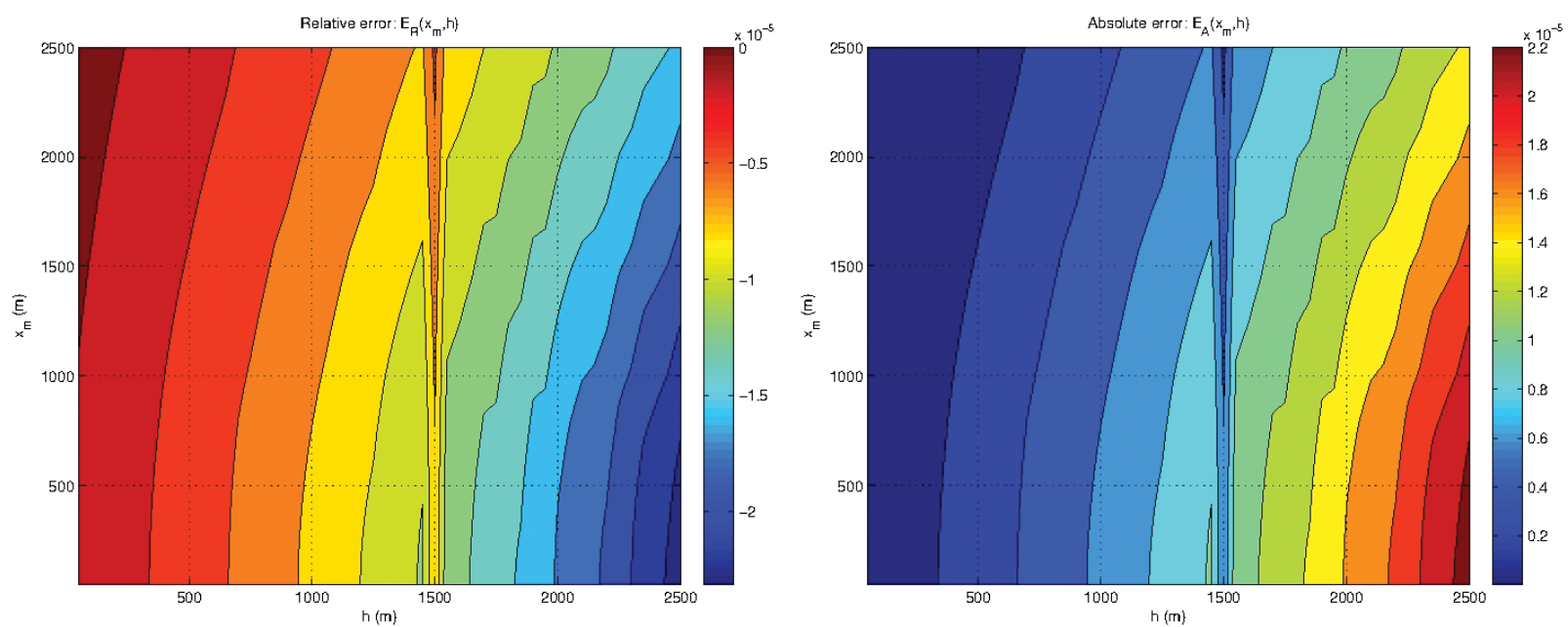

Figure 14 - Contour maps of the relative error calculated by Eq. (12) (left), and of the absolute error calculated by Eq. (13) (right), showing valleys of minimum around the nominal value at the center of figures.

The $\ln \mathbf{m}$ means the vector of the logarithms of the elements of $\mathbf{m}$, therefore

$$
\partial \ln \mathbf{m}=\left[\partial m_{1} / m_{1} m_{2} / m_{2} \ldots m_{M} / m_{M}\right]^{T} .
$$

The $i^{\text {th }}$ element of $\bar{S}_{i j}$ is defined by:

$$
\left.\bar{S}_{i j} \triangleq \frac{\partial t_{i}(\mathbf{m}) / t_{i}}{\partial m_{j} / m_{j}}\right|_{m_{0}}=S_{i j} \frac{m_{0 j}}{t_{0 i}}
$$

where $S_{i j}$ is the $i j^{\text {th }}$ element of absolute sensitivity function $\mathbf{S}$ of Eq. (20). The $i^{\text {th }}$ element of the relative error of the system function is defined by:

$$
\frac{\Delta t_{i}}{t_{0 i}} \triangleq \sum_{j=1}^{j=M} S_{i j} \frac{\Delta m_{j}}{m_{0 j}},
$$

and the maximum Relative Error of the system function by

$$
\left|\frac{\Delta t_{i}}{t_{0 i}}\right| \triangleq \sum_{j=1}^{j=M}\left|S_{i j}\right|\left|\frac{\Delta m_{j}}{m_{0 j}}\right| .
$$

There are also two ways to define a semirelative sensitivity function as follows.

The Upper-semirelative Logarithmic sensitivity function defined by:

$$
\left.\check{S}_{i j} \triangleq \frac{\partial \ln t_{i}(\mathbf{m})}{\partial m_{j}}\right|_{\mathbf{m}_{0}},
$$

with the components $\check{S}_{i j}$ by

$$
\left.\check{S}_{i j} \triangleq \frac{\partial t_{i}(\mathbf{m}) / t_{i}}{\partial m_{j}}\right|_{\mathbf{m}_{0}}=\frac{1}{t_{0 i}} S_{i j} .
$$

The Lower-semirelative Logarithmic sensitivity function defined by:

$$
\left.\hat{S}_{i j} \triangleq \frac{\partial t_{i}(\mathbf{m})}{\partial \ln m_{j}}\right|_{\mathbf{m}_{0}}
$$

and the components $\hat{S}_{i j}$, in terms of $S_{i j}$, by

$$
\left.\hat{S}_{i j} \triangleq \frac{\partial t_{i}(\mathbf{m})}{\partial m_{j} / m_{j}}\right|_{\mathbf{m}_{0}}=m_{0 j} S_{i j} .
$$

For this measure, the $i^{\text {th }}$ element of the relative error of the system function is defined in terms of $S_{i j}$ by:

$$
\Delta t_{i} \triangleq \sum_{j=1}^{j=M} S_{i j} \frac{\Delta m_{j}}{m_{0 j}},
$$

and the absolute error by

$$
\left|\Delta t_{i}\right| \triangleq \sum_{j=1}^{j=M}\left|S_{i j}\right|\left|\frac{\Delta m_{j}}{m_{0 j}}\right| .
$$

Due to the mathematical representation used for the sensitivity functions, $S_{i, j}$, and for the derivatives, $G_{i, j}$, we have the equality $S_{i, j}=G_{i, j}$, where the necessary functions $G_{i, j}$ are given in Appendix C. Properties of the function $G_{i, j}$ is given by Eq. (41).

\section{Appendix B: The CRSGM}

As a simple description for completion of the text for the optimization fundamentals, it is desired with the Price method a solution of the global non-constrained nonlinear optimization problem, structured in the following form: $\mathbf{m i n} \chi(\mathbf{m}), \mathbf{m} \in R^{M}$, where $\chi: R^{M} \rightarrow R$ is a continuous function; that is, a minimum $\chi(\mathbf{m})$ of the continuous function is searched, where the 
parameter vector $\mathbf{m}$ (dimension $\mathrm{M}$ ) to be determined is defined in the $R^{M}$ space. In this form, $\mathbf{m}$ represents point-coordinates $m_{i},(i=1, M)$ in the continuous parameter space. The function object of minimizations is considered multimodal.

To initiate the process, a $V$ search domain is defined through the specification of explicit constraints to each parameter. Next, at each iteration $k$, it is defined a predetermined quantity, $N$, of test points randomly chosen in $V$ and consistent with the constraints (in case they are imposed) forming the set:

$$
B_{1}^{k}=\left\{\mathbf{m}_{\mathbf{1}}{ }^{(k)}, \mathbf{m}_{\mathbf{2}}{ }^{(k)}, \mathbf{m}_{\mathbf{3}}{ }^{(k)}, \ldots, \mathbf{m}_{\mathbf{N}}{ }^{(k)}\right\} .
$$

The functional $\chi(\mathbf{m})$ is evaluated at all $N$ points, and the position and value of the function $\chi(\mathbf{m})$ are saved in a matrix $\mathbf{A}$ $[N \times(M+1)]$.

At each iteration $k$, a new test point $P, \widehat{\mathbf{m}}^{(k)}$, is calculated using a random sub-set $B_{2}^{(k)}$ of $B^{(k)}$ described in the form:

$$
\widehat{\mathbf{m}}^{(k)}=\mathbf{c}^{(k)}-\left(\mathbf{m}_{20}^{(k)}-\mathbf{c}^{(k)}\right),
$$

being $\mathbf{c}$ the centroid defined by:

$$
c_{j}^{(k)}=\frac{1}{M} \sum_{i=1}^{M} m_{2 i}^{(k)} \quad(j=1, M) .
$$

Next, a test is done if the point $P$ satisfies the constraints, and if

$$
\chi\left(\widehat{\mathbf{m}}^{(k)}\right)<\chi\left(\widehat{\mathbf{m}}_{\max }^{(k)}\right) .
$$

In case these conditions are not satisfied, the process returns for new definitions. The probability that the points converge to the global minimum depends on the distribution, on the value of $N$, on the complexity of the functional, in the nature of constraints and in the choice of the test points.

\section{Appendix C: The GM}

The Taylor series expansion of the function $\mathbf{t}\left(x_{m}, h ; \mathbf{m}_{\mathbf{2}}\right)$, in the neighborhood of $\mathbf{t}\left(x_{m}, h ; \mathbf{m}_{\mathbf{1}}\right)$, to the first order is written as an approximation ( $\approx)$ as:

$$
\begin{gathered}
t_{i}\left(x_{m}, h ; \mathbf{m}_{\mathbf{2}}\right) \approx t_{i}\left(x_{m}, h ; \mathbf{m}_{1}\right) \\
+\sum_{j=1}^{M} \frac{\partial t_{i}}{\partial m_{j}}\left(x_{m}, h ; \mathbf{m}_{\mathbf{1}}\right) \Delta m_{j}, \quad(i=1, N) .
\end{gathered}
$$

The observed data is represented by $t_{i}^{(\mathrm{obs})}\left(x_{m}, h ; \mathbf{m}_{2}=\right.$ true), and the predicted data by $t_{i}^{\text {(pre) }}\left(x_{m}, h ; \mathbf{m}_{1}=\right.$ model $)$. From these definitions, with $\mathbf{m}_{\mathbf{1}}=\mathbf{m}$, we can write Eq. (37) as:

$$
\begin{aligned}
& t_{i}^{\text {(obs) }}\left(x_{m}, h\right)-t_{i}^{\text {(pre) }}\left(x_{m}, h ; \mathbf{m}\right) \\
& =\sum_{j=1}^{M} \frac{\partial t_{i}^{\text {(pre })}}{\partial m_{j}}\left(x_{m}, h ; \mathbf{m}\right) \Delta m_{j},
\end{aligned}
$$

and in the matrix compact form

$$
\Delta \mathbf{t}\left(x_{m}, h ; \mathbf{m}\right)=\mathbf{G}\left(x_{m}, h ; \mathbf{m}\right) \Delta \mathbf{m} .
$$

This equation represents a linearized form to obtain a solution to the nonlinear problem, where $\Delta \mathbf{t}\left(x_{m}, h ; \mathbf{m}\right)$ is a column vector, $(N \times 1)$, that represents the data deviations; $\Delta \mathbf{m}$ is a column vector, $(M \times 1)$, that represents the parameter deviations; and $\mathbf{G}\left(x_{m}, h ; \mathbf{m}\right)$ is the problem matrix, $(N \times M)$, that has the data information along the columns, the parameter information along the lines, and the elements $G_{i, j}$ are given by the partial derivatives of $t\left(x_{m}, h ; \mathbf{m}\right)$ in the form:

$$
G_{i, j}=\frac{\partial t_{i}^{\text {(pre) }}\left(x_{m}, h ; \mathbf{m}\right)}{\partial m_{j}}, \quad(i=1, N ; j=1, M) .
$$

These partial derivatives are rather long, and they are used to represent the sensitivity functions with respect to the parameters.

The derivatives $G=G\left(x_{m}, h ; \mathbf{m}\right)$, described below, have a general form of the type:

$$
\begin{gathered}
G\left(x_{m}, h ; \mathbf{m}\right)= \\
\frac{a(\mathbf{m})\left(x_{\mathbf{m}}-x_{0}\right)+b(\mathbf{m})\left(x_{\mathbf{m}}-x_{0}\right)^{2}+d(\mathbf{m}) h^{2}}{t\left(x_{m}, x_{0} ; \mathbf{m}\right)}
\end{gathered}
$$

that would rapidly decay with respect to $t\left(x_{m}, h ; \mathbf{m}\right)$, and increase with respect to the numerator function controlled by the coefficients, $[a(\mathbf{m}), b(\mathbf{m}), d(\mathbf{m})]$, and sign of the $\left(x_{\mathbf{m}}-x_{0}\right)^{1}$ factor.

The continuous partial derivative with respect to $\alpha_{0}$, and shown in Figure 10, is given by:

$$
\frac{\partial t\left(x_{m}, h\right)}{\partial \alpha_{0}}=\frac{\frac{2 \cos \alpha_{0}\left(x_{m}-x_{0}\right)}{v_{0}}\left(t_{0}+\frac{2 \sin \alpha_{0}\left(x_{m}-x_{0}\right)}{v_{0}}\right)-\frac{2 t_{0} \cos \alpha_{0} \sin \alpha_{0}}{v_{0}}\left(\frac{\left(x_{m}-x_{0}\right)^{2}}{R_{\mathrm{N}}}+\frac{h^{2}}{R_{\mathrm{NP}}}\right)}{\sqrt{\left(t_{0}+\frac{2 \sin \alpha_{0}\left(x_{m}-x_{0}\right)}{v_{0}}\right)^{2}+\frac{2 t_{0} \cos ^{2} \alpha_{0}}{v_{0}}\left(\frac{\left(x_{m}-x_{0}\right)^{2}}{R_{\mathrm{N}}}+\frac{h^{2}}{R_{\mathrm{NP}}}\right)}} .
$$


The continuous partial derivative with respect to $R_{\mathrm{NIP}}$, and shown in Figure 11, is given by:

$$
\frac{\partial t\left(x_{m}, h\right)}{\partial R_{\mathrm{NP}}}=-\frac{\frac{t_{0} h^{2} \cos ^{2} \alpha_{0}}{v_{0} R_{\mathrm{NP}}^{2}}}{\sqrt{\left(t_{0}+\frac{2 \sin \alpha_{0}\left(x_{m}-x_{0}\right)}{v_{0}}\right)^{2}+\frac{2 t_{0} \cos ^{2} \alpha_{0}}{v_{0}}\left(\frac{\left(x_{m}-x_{0}\right)^{2}}{R_{\mathrm{N}}}+\frac{h^{2}}{R_{\mathrm{NIP}}}\right)}} .
$$

The continuous partial derivative with respect to $R_{\mathrm{N}}$, and shown in Figure 12, is given by:

$$
\frac{\partial t\left(x_{m}, h\right)}{R_{\mathrm{N}}}=-\frac{\frac{t_{0}\left(x_{m}-x_{0}\right)^{2} \cos ^{2} \alpha_{0}}{v_{0} R_{\mathrm{N}}^{2}}}{\sqrt{\left(t_{0}+\frac{2 \sin \alpha_{0}\left(x_{m}-x_{0}\right)}{v_{0}}\right)^{2}+\frac{2 t_{0} \cos ^{2} \alpha_{0}}{v_{0}}\left(\frac{\left(x_{m}-x_{0}\right)^{2}}{R_{\mathrm{N}}}+\frac{h^{2}}{R_{\mathrm{NIP}}}\right)}} .
$$

The continuous partial derivative with respect to $v_{0}$, and shown in Figure 13, is given by:

$$
\frac{\partial t\left(x_{m}, h\right)}{\partial v_{0}}=-\frac{\frac{2 \sin \alpha_{0}\left(x_{m}-x_{0}\right)}{v_{0}^{2}}\left(t_{0}+\frac{2 \sin \alpha_{0}\left(x_{m}-x_{0}\right)}{v_{0}}\right)+\frac{t_{0} \cos ^{2} \alpha_{0}}{v_{0}^{2}}\left(\frac{\left(x_{m}-x_{0}\right)^{2}}{R_{\mathrm{N}}}+\frac{h^{2}}{R_{\mathrm{NP}}}\right)}{\sqrt{\left(t_{0}+\frac{2 \sin \alpha_{0}\left(x_{m}-x_{0}\right)}{v_{0}}\right)^{2}+\frac{2 t_{0} \cos ^{2} \alpha_{0}}{v_{0}}\left(\frac{\left(x_{m}-x_{0}\right)^{2}}{R_{\mathrm{N}}}+\frac{h^{2}}{R_{\mathrm{NP}}}\right)}} .
$$

From these partial derivatives, the quantities $\bar{S}_{i j}\left(\mathbf{m}_{0}\right)=\frac{\partial \ln t_{i}\left(\mathbf{m}_{\mathbf{0}}\right)}{\partial \ln m_{0 j}}=\frac{m_{0 j}}{t_{0 i}} S_{i j}\left(\mathbf{m}_{0}\right)$ are calculated.

\section{ACKNOWLEDGMENTS}

The authors would like first to thank the paper Reviewers for their patience and positive contributions. We would like to thank the Brazilian institutions FINEP (Financiadora de Estudos e Projetos) Fase 5, PETROBRAS (Petróleo Brasileiro S/A) for the research support, and in special to the project National Institute of Science and Technology (Instituto Nacional de Ciência e tecnologia, INCT-GP, of MCT/CNPq/FINEP). The thanks are also extended to CAPES (Coordenação de Aperfeiçoamento de Pessoal de Nível Superior) for the scholarship, and to the Wave Inversion Technology Group for the academic cooperation support.

\section{REFERENCES}

BERNABINI M, CARRIONP, JACOVITTIG, ROCCA F, TREITEL S \& WORTHINGTON M. 1987. Deconvolution and inversion. Blackwell Scientific Publications. 355 pp.

BRACHETTI P, CICCOLI MDF, PILLO GD \& LUCIDI S. 1997. A new version of Price's algorithm for global optimization. Journal of Global Optimization, pages 165-184.

DUVENECK D. 2004. Tomographic determination of seismic velocity models with kinematic wavefield attributes. Ph.D. thesis, Universität Fridericiana Karlsruhe. 181 pp.

FLETCHER R. 2001. Practical methods of optimization. John Wiley and Sons. $436 \mathrm{pp}$.
FOMEL S \& KAZINNIK R. 2010. Non-hyperbolic common reflection surface. In: 79th Annual International Meeting of the Society of Exploration Geophysics (CD-ROM), 1(1): 1-6

FRANK PM. 1978. Introduction to system sensitivity theory. Academic Press. $386 \mathrm{pp}$

MANN J. 2002. Extensions and applications of the common-reflectionsurface stack method. Ph.D. thesis, Universität Fridericiana Karlsruhe. $165 \mathrm{pp}$.

MAUCH R. 1999. Coherency analysis of seismic data. Master's thesis, Universität Fridericiana Karlsruhe. 120 pp.

MENKE W. 2002. Geophysical data analysis. Discrete inverse theory. Academic Press. 289 pp.

MULLER T. 1999. The common reflection surface stack - seismic imaging without explicit knowledge of the velocity model. Ph.D. thesis, Universität Fridericiana Karlsruhe. 139 pp.

PRICE W. 1983. Global optimization by controlled random search. Journal of Optimization Theory and Applications, 40(3): 333-348.

SALTELLI A, TARANTOLAS, CAMPOLONGO F \& RATTO M. 2004. Sensitivity analysis in practice. A guide to assessing scientific models. John Wiley and Sons. 219 pp.

TARANTOLAA. 1984. Inverse problem theory. SIAM. 342 pp.

TOLDI JL. 1985. Velocity analysis without picking. Ph.D. thesis, Stanford University. 181 pp. 
VIEIRA W \& LEITE L. 2009. Sensitivity and resolution of the CRS stack operator (in portuguese). Workshop of the Exploration Geophysics Network. Salvador, Bahia, Brazil. CD-ROM.
VIEIRA W, LEITE L \& NUNES FSM. 2011. Automatic picking in the semblance domain. In: 12th International Congress of the Brazilian Geophysical Society. Rio de Janeiro, Brazil. CD-ROM.

Recebido em 01 março, 2013 / Aceito em 20 dezembro, 2013

Received on March 01, 2013 / Accepted on December 20, 2013

\section{NOTES ABOUT THE AUTHORS}

Lourenildo W.B. Leite is a professor of Geophysics in the Undergraduate and Graduate Courses of the Federal University of Pará (UFPA), Brazil. He obtained a Masters and a Doctorate in Geophysics from Saint Louis University, Missouri, USA. He develops research work in applied seismic for oil and gas exploration aiming at velocity analysis, stack, inversion, migration, and attribute analysis. He is a member of the SBGF, SEG, EAGE and SSA scientific societies.

Wildney W.S. Vieira is a Doctorate student at the Graduate Course in Geophysics of the Federal University of Pará (UFPA), Brazil. He obtained a Bachelors in Mathematics (in 2007), and a Masters of Geophysics (in 2011), both degrees at UFPA. He develops research work in inversion techniques for seismic applied to oil and gas exploration. His area of interest has been ultimately focused at imaging by a complete processing workflow passing by data quality control, velocity analysis, inversion and migration. He is a member of the SBGF scientific society. 